\title{
Interference of the profiles when meshing internal straight splines with gear shapers
}

\author{
Svetlin Marinov, Ognyan Alipiev, and Toni Uzunov \\ Department of Theory of Mechanisms and Machines, University of Ruse, Ruse 7017, Bulgaria
}

\begin{abstract}
The possibilities for cutting internal straight splines with gear shapers by internal meshing, without interference between meshed profiles, are shown in this article. The interference is an undesirable occurrence whereby the straight profile of the splines and the instrumental contour intersect with each other beyond the meshing line. As a result, the shaper teeth cut off a part of the splines' straight profile and they are made with some defects. The research shows that the undesirable interference depends directly on the parameters of the splined opening and the number of shaper teeth. Also, it is determined that the interference can be avoided by reducing the number of shaper teeth and the height of the splines. Furthermore, the maximum number of shaper teeth for all standard splines for which the interference is absent, are defined in the article. The results are confirmed by computer simulations of the corresponding instrumental meshing.
\end{abstract}

\section{Introduction}

Nowadays, for cutting internal splines in industrial manufacturing, two completely different traditional methods have been established: 1) single cutting of every spline with simple cutter by interrupted detachment of the blank $[1,2] ; 2)$ cutting all splines at the same time (broaching) [3]. Both of these methods belong to the so called "form- copying methods".

In the last years, for mass producing of splines parts, other methods, working without cutting the material, have been proposed. In these methods, the splines are made by extrusion [4], stamping [5], plastic deformation [6] and other process [7].

Internal straight splines can be also generated using the presented by the authors' non-traditional 'generating method" [8, 9]. In this method, an internal meshing between the cutting tool (a shaper gear) and the splines is imitated. It is similar to the well-known method that is used in practice for internal involute spur gear manufacturing [10]. Based on the authors' theoretical research, in [8], the parametric equations of the theoretical meshing line and the gear shaper teeth contour have been determined. The effect of the number of shaper teeth over their contour has been explored. It is theoretically proven that if the gear shaper number of teeth is twice as small as that of the splines, the profile of the tool teeth is significantly simplified. In that case, the profile transforms to a part of a circle. It has also been found that when the shaper has only a few teeth, their profile contour is a broken curve. In this regard, a limiting condition for avoiding this curvature and a formula for determining the minimum number of gear shaper teeth have been defined.
When the number of the shaper teeth increases over certain value, there is a possibility for undesirable intersection between the cutting contours and the straight profiles of the splines. In the gearing theory, this occurrence is known as "interference" of the teeth profiles. The interference, as well the undercutting of the profiles [11] and the teeth sharpening [12, 13], is destructive occurrences. In the internal instrumental meshing, two types of interference are possible, depending on the motions of the gear shaper and the splines.

The first type, defined as "radial interference" [14, 15], may occur while the gear shaper is radially incised within the splines that are being formed. In this case, the tool is radially moved against the splines, whereby the center distance of the gear meshing gradually increases to reach the required value. Two cases are possible: 1) only radial incision of the shaper within the splines; 2) simultaneous radial incision and a synchronous rotation of the shaper and the splines.

The second type, defined as "axial interference" [14] or "second class interference" [15] may occur when the gear shaper and the splines make only synchronous rotating motions. Then, the axes of the splined opening and the gear shaper stay on the set center distance of the gear meshing. Because the second interference exists at synchronous rotation of the two gears, the authors propose the term "generating interference" to be used instead of "axial" or "second class" interference.

"The radial interference" and "the generating interference" usually can be avoided by decreasing the number of the gear shaper teeth. This means that, for correctly forming of the splines, it is necessary to define the maximum number of shaper teeth in advance, in which the interferences, mentioned above, do not exist. 


\section{Generating interference}

The essence of the interference in meshing becomes clear by Fig. 1 and Fig. 2. Fig. 1 shows the instrumental meshing between the gear shaper and the internal straight splines when the interference is absent and in Fig. 2 a case with generating interference between teeth contours is visualized.

In the shown figures, a clockwise direction for the rotation of the tool and splines is chosen. So, the first contact of the shaper teeth with the opening of the piece (with radius $r$ ) is in point $C$, in which the teeth profiles are in position 1 . While the profiles are rotating from position 1 to position 3 , the shaper contour is cutting material, but is not forming the straight profile of the spline. The zone from position 1 to position 3 is defined as "generating incision zone". Position 3 is beginning of "the gear meshing" in which the shaper teeth contour is contacting (gearing) with the innermost point of the splines straight profile. This position is found by point $F$, which is a cross point between the theoretical meshing line $B P A$ and the splines inner circle with radius $r$. The final generation of the splines straight profile is after shaper teeth and splines have been rotated from position 3 to position 4. This stage of the splines cutting is defined as "actual instrumental meshing" in which, all contact points between meshed profiles lay on the actual meshing line FPE. Position 4 is the "end of the gear meshing" and after that, the shaper teeth and the straight profile of the spline discontinue the meshing. The location of the meshed profiles in position 4 is determined from the meshing line end point $E$. In this case, the point $E$ is found as a cross point of the theoretical meshing line $B P A$ and the shaper outer circle with radius $r_{a}$. In the instrumental meshing, the final point $\mathrm{A}$ from the theoretical meshing line is found as a cross point between the splines outer circle $R$ and the vertical line placed at distance $b / 2$ from the meshing pole $P$.

The generating interference may occur after rotating the profiles from position 4 to position 8 (Fig. 1 and Fig. 2). After that, the contact between the shaper tooth and the spline is not possible. The position 8 is the end position of the possible interference, defined by point $D$. It is found as a cross point between the shaper outer circle $r_{a}$ and the splines inner circle $r$. According to the set geometric parameters, after position 4, the tooth profile of the shaper cutter may cross or not the spline straight profile. In this connection, the generating interference is defined as an undesirable intersection between the shaper teeth profiles and the splines straight profiles, realized out of the actual meshing line FPE.

In Fig. 1 it is shown that in positions 6 and 8 , the shaper tooth contour does not touch or cross the spline straight profile, and the positions 6 and 8 are located after position 4 , i.e. after the actual meshing zone. This means that in the instrumental meshing shown in Fig. 1 there is no interference.

The interference between the instrumental and cut profiles exists in the positions 5, 6, 7 and 8, shown in Fig. 2, from which it is clear that the shaper tooth crosses the spline straight profile. In practice, this leads to an



Fig. 1. Instrumental meshing with no interference. 


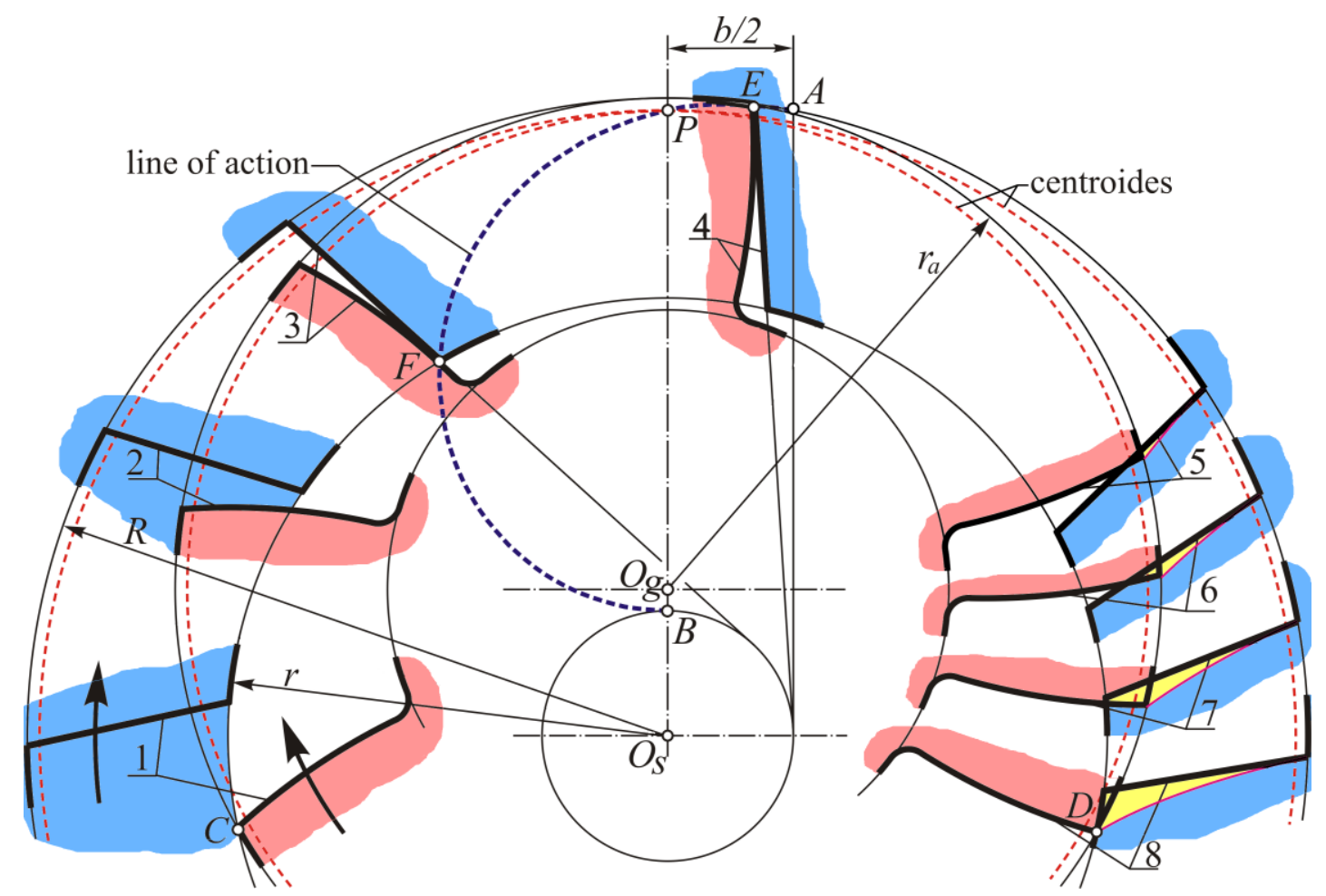

Fig. 2. Instrumental meshing with interference.

undesirable cut of the spline profile, which is generated in instrumental meshing from position 3 to position 4 . A characteristic feature for this case is that the cutting off from the straight profile of the spline is increasing from position 5 to position 8 , where the interference is the greatest.

\section{Main parameters of the splines and the instrumental meshing}

The problem for defining the interference between the gear shaper and the splines in their synchronous rotation (Fig. 3) is usually solved after defining the main parameters of the internal spline:

$z$ - number of splines;

$b$ - width of the splines;

$R$ - radius of the splines outer circle;

$r$ - radius of the splines inner circle;

and the respective geometric parameters of the gear instrumental meshing:

$R_{S}$ - radius of the splines centroidal circle;

$R_{g}$ - shaper centroidal radius

$$
R_{g}=\frac{z_{g}}{z} R_{S},
$$

where $z_{g}$ is the number of shaper teeth;

$a$ - center distance in instrumental meshing

$$
a=R_{S}-R_{g}=\frac{z-z g}{z} R_{S} .
$$

After setting the main parameters of the splines, their centroidal radius $R_{S}$ and the radius of the gear shaper outer circle $r_{a}$ are defined. Usually, in centroidal cutting of external or internal splines, $R_{S}$ is chosen equal to minimum possible radius $R_{s, \text { min }}$ (Fig. 3), which is calculated by equation (3)

$$
R_{s}=R_{s, \min }=\sqrt{R^{2}-(b / 2)^{2}},
$$

and the radius $r a$ is defined by the equation

$$
r_{a}=R-a,
$$

which ensures touching the circles with radiuses $R$ and $r a$ in point $C$.

Having in mind the equations (2), (3) and (4), for the radius of the shaper outer circle we finally have

$$
r a=R-\frac{z-z_{g}}{z} \sqrt{R^{2}-(b / 2)^{2}} .
$$

The straight splines profile generation, using the parameters defined above, becomes clear by actual instrumental meshing shown in Fig. 3a, where the line $F K P E$ is an actual meshing line. In the start point $F$ of this line is formed the inner point $M$ from the spline while it is meshing with point $H$ from the profile $g$ of the shaper tooth. After that, by corresponding points $K$ between the profiles $g$ and $s$ are successively generated all points from the spline straight profile $M N$. The instrumental meshing ends in the end point $E$ from the actual meshing line, where the point $G$ from the shaper tooth is forming the point $N$ from the spline (the points $G$ and $N$ are meshed in point $E$ ). After this position, while the shaper and the splines are rotating, the point $G$ is generating the transition curve $N L$ of the spline (Fig. 3b). After that, the profiles $g$ and $s$ do not contact with each other if there is no interference. If there is interference, the profiles $g$ and $s$ are crossed. 


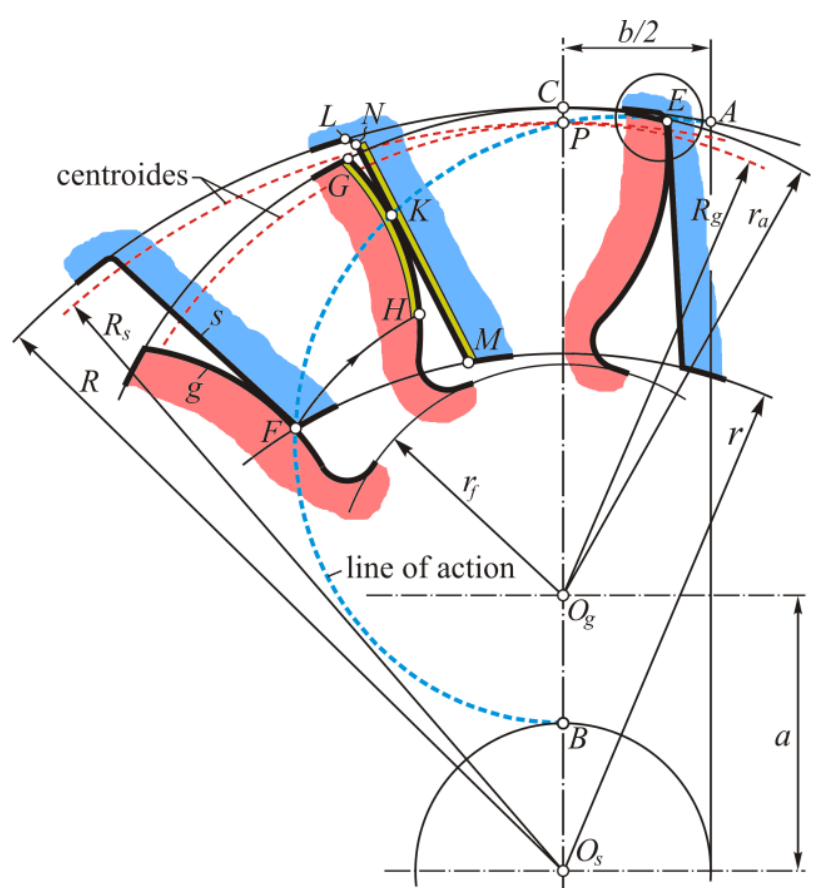

a)

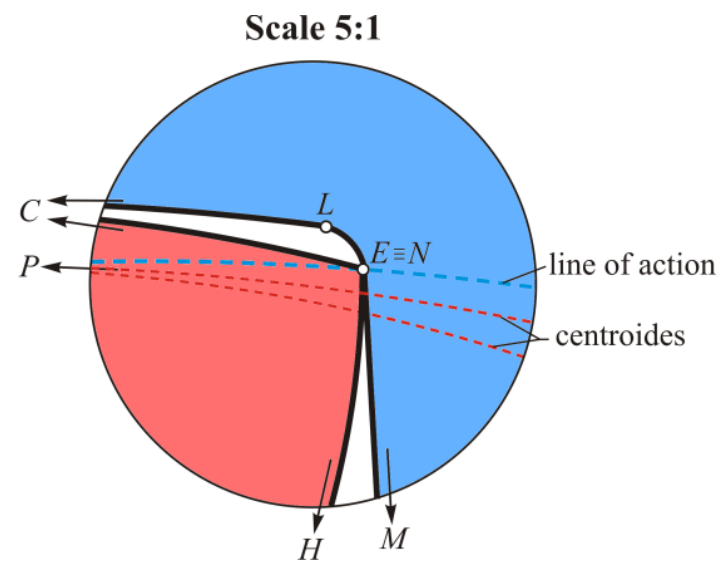

b)

Fig. 3. Instrumental meshing.

The problem, related to defining the possible interference is solved by finding the relative positions of the shaper tooth and the straight spline in the zone after actual instrumental meshing. This zone starts after the final generation of the straight profile $M N$ and ends in the position where the shaper tooth comes out of the spline. In the considered case it is appropriate the zone between the positions 4 and 8, shown in Fig. 1 and Fig. 2 , to be defined as "zone of possible interference".

\section{Geometric modeling of the interference at $z_{g} \neq z / 2$}

\subsection{Requirement for avoiding the interference}

The generating interference can be clarified by Fig. 4, showing the beginning and the end position of the teeth profiles in the zone of possible interference. In the first position, the peak point of the shaper tooth coincides with the end point $E$ from the actual meshing line $F P E$. Distinctive mark for the position shown of the teeth profiles is the location of the point $D$ from the shaper tooth, which lies on the splines inner circle $r$. From the Fig.4 it is visible that the generating interference is absent when the equation (6) is fulfilled

$$
\alpha \geq \beta \text {, }
$$

where $\alpha$ is the rotation angle of the splines in the zone of possible interference, defined as boundary angle of the straight splines. Respectively, the angle $\beta$ defines the end position of the shaper tooth is called boundary angle of the gear shaper.

Having in mind that

$$
\alpha-\beta=\mu,
$$

the requirement for avoiding the generating interference can be formulated as

$$
\mu \geq 0
$$

when $\mu$ is defined as interference angle.

\subsection{Boundary angle of the straight splines}

In the instrumental meshing, the splines to be cut and the gear shaper rotate synchronously in ratio depending on splines number $z$ and the number of the shaper teeth. Thus the boundary angle of the straight splines is defined by:

$$
\alpha=\alpha_{g} z_{g} / z,
$$

In equation (9), $\alpha_{g}$ is the rotation angle of the gear shaper from the beginning to the end of its position in the zone of possible interference.

From Fig. 4 it is clear that

$$
\alpha_{g}=\theta_{g}-\gamma_{g},
$$

where

$$
\theta_{g}=\pi-\eta
$$

Furthermore

$$
\cos \theta_{g}=-\cos \left(\pi-\theta_{g}\right)=-\cos \eta,
$$

and for the triangle $\mathrm{DO}_{g} \mathrm{O}_{s}$

$$
r^{2}=r_{a}^{2}+a^{2}-2 r_{a} a \cos \eta
$$

which, by equation (12), transforms into

$$
\theta_{g}=\arccos \left(\frac{r^{2}-r_{a}^{2}-a^{2}}{2 r_{a} a}\right) \text {. }
$$

The angle $\gamma_{g}$ is defined by the scaled right-angled triangle $\mathrm{EBO}_{g}$ in Fig. 4.

$$
\gamma_{g}=\arcsin \left(\frac{X_{E}}{r_{a}}\right),
$$

and after that, the shaper rotating angle $\alpha_{g}$ is defined.

Further, after the equations (14) and (15) are substituted in equation (10), the boundary angle of the straight splines is determined by

$$
\alpha=\left[\arccos \left(\frac{r^{2}-r_{a}^{2}-a^{2}}{2 r_{a} a}\right)-\arcsin \left(\frac{X_{E}}{r_{a}}\right)\right] \frac{z_{g}}{z},
$$




\section{Scale 3:1}

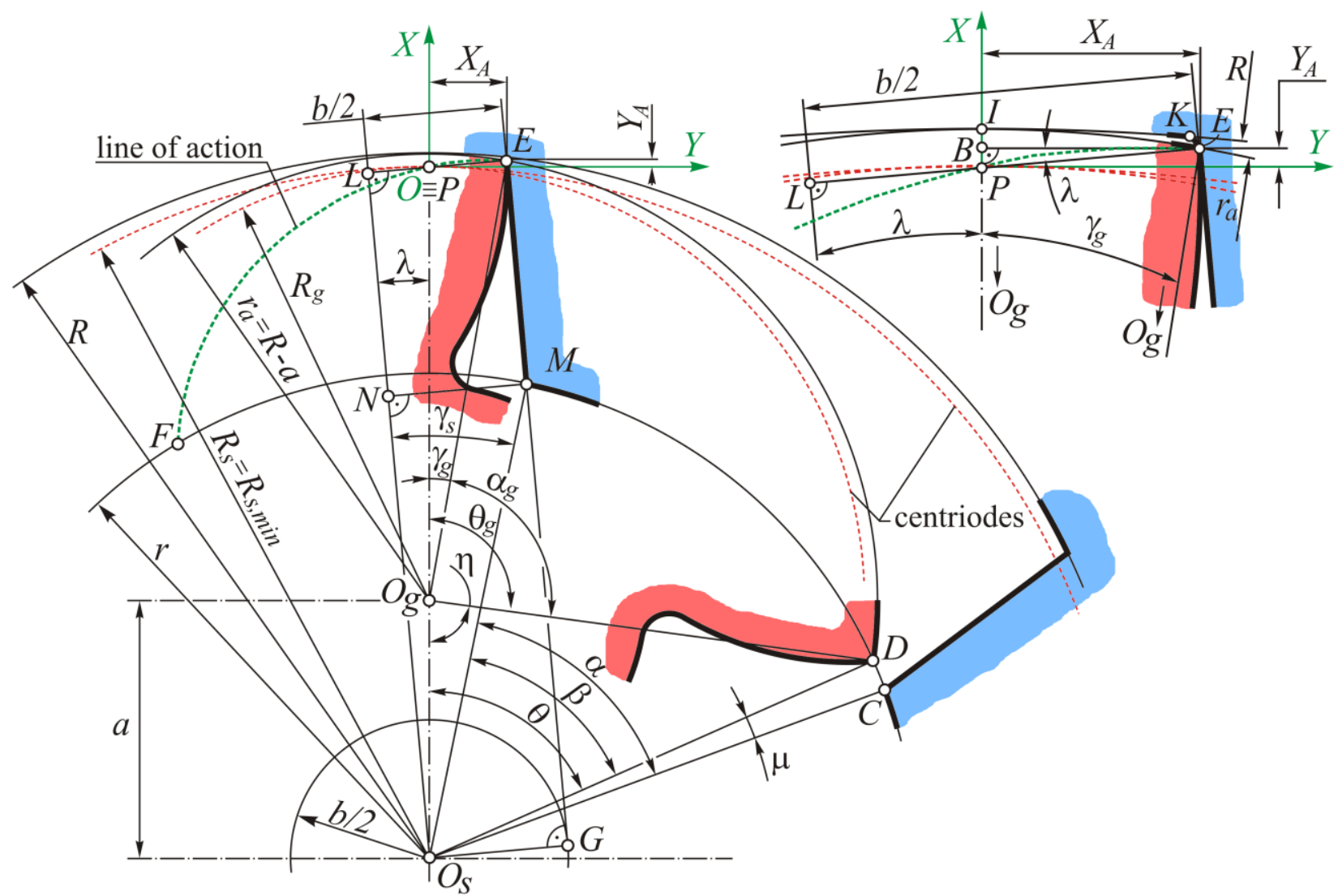

Fig. 4. Teeth profiles position in the interference zone.

in which the abscissa $X_{E}$ of the point $E$ from the meshing line in coordinate system $X O Y$ is defined by numerical solution, clarified in 4.5

\subsection{Boundary angle of the gear shaper}

The boundary angle of the shaper $\beta$, as it shown in Fig 4 , depends directly on angles $\theta, \lambda$ and $\gamma_{s}$. They are connected by equation

$$
\beta=\theta+\lambda-\gamma_{s} .
$$

For defining the angle $\theta$ the law of cosines is used, applied for the triangle $\mathrm{DO}_{g} \mathrm{O}_{s}$

$$
\cos \theta=\frac{r^{2}-r_{a}^{2}+a^{2}}{2 r a}
$$

The angle $\lambda$ is defined from the scaled right-angled triangle $E B P$ in Fig 4

$$
\tan \lambda=\frac{Y_{E}}{X_{E}},
$$

and from the triangle $M N O_{s}$ by equation

$$
\sin \gamma_{s}=\frac{b}{2 r},
$$

the angle $\gamma_{s}$ is defined, which determines the position of point $M$.
After the equations (18), (19) and (20) are substituted in (17), for the boundary angle of the gear shaper we finally have the equation

$\beta=\arccos \left(\frac{r^{2}-r_{a}^{2}+a^{2}}{2 r a}\right)+\operatorname{arctg}\left(\frac{Y_{E}}{X_{E}}\right)-\arcsin \left(\frac{b}{2 r}\right)$,

in which $X_{E}$ and $Y_{E}$ are the point $E$ coordinates from the meshing line.

\subsection{Interference angle and level of interference}

The relation between the angles $\theta_{g}, \gamma_{g}, \theta, \lambda, \gamma_{s}$ and the interference angle $\mu$ showed in Fig. 4 , is clarified by the equation

$$
\mu=\left(\theta_{g}-\gamma_{g}\right) \frac{z g}{z}-\theta-\lambda+\gamma_{s}
$$

The final equation for the interference angle is defined after substitution of equations (16) and (21) in (7). Then the $\mu$ equation is

$$
\begin{aligned}
& \mu=\left[\arccos \left(\frac{r^{2}-r_{a}^{2}-a^{2}}{2 r_{a} a}\right)-\arcsin \left(\frac{X_{E}}{r_{a}}\right)\right] \frac{z_{g}}{z}- \\
& -\arccos \left(\frac{r^{2}-r_{a}^{2}+a^{2}}{2 r a}\right)-\operatorname{arctg}\left(\frac{Y_{E}}{X_{E}}\right)+\arcsin \left(\frac{b}{2 r}\right)
\end{aligned}
$$


If the value from equation (23) is positive number, there is no interference between the cutting contours and the cut straight spline. The interference appears when the value of $\mu$ is negative number. Also, the interference increases with the increasing of the absolute value of $\mu$.

For the determination of the largest part that is cut off from the straight spline profile, when interference is present, using the term "level of interference" is more appropriate. The level of interference $\Delta$ is defined as a length of the arc $C D$ which is cut off from the spline width in the circle with radius $r$. It is calculated by

$$
\Delta=\mu r
$$

where $\Delta$ is a negative number (measured in $\mathrm{mm}$ ) when an interference is occurred, and a positive number when there is no interference.

\subsection{Numerical solution of the meshing line end point coordinates}

The coordinates $X_{E}$ and $Y_{E}$ of the end point $E$ from the meshing line FPE (Fig. 4), which help to calculate the equations (16), (21) and (23), can be defined after solving the system (25) with three equations with three variables:

$$
\begin{aligned}
& X_{E}=\left(\frac{b}{2}-R_{S} \sin \psi\right) \cos \psi \\
& Y_{E}=\left(\frac{b}{2}-R_{S} \sin \psi\right) \sin \psi \\
& X_{E}^{2}+\left(Y_{E}+R_{g}\right)^{2}=r_{a}^{2} .
\end{aligned}
$$

The three variables are the coordinates $X_{E}, Y_{E}$ and the angular parameter $\psi$ of the point $E$. In that, the first two are the meshing line parametric equations [8], and the third one is the shaper outer circle $r_{a}$ equation in the coordinate system $X O Y$ (Fig. 4).

The determination of $\psi, X_{E}$ and $Y_{E}$ from the system (25) is performed by numerical solution, which can be done by the algorithm described below, appropriate for computer program Microsoft Excel:

1) The splines' main parameters $b, R, z$ are set and after that the spline centroidal radius $R_{s}$ is defined.

2) The number of the shaper teeth is chosen and using the equations (1) and (5) the shaper centroidal radius $R_{g}$ and outer circle radius $r_{a}$ are defined. The radius $r_{a}$ is main parameter in this numerical solution.

3) A current value of the angular parameter $\psi_{i}$ is set, and from the first two equations from the system, the current values of the coordinate $X_{E i}$ and $Y_{E i}$ are calculated. The initial value of the angular parameter is $\psi_{i}=\psi_{0}=0$.

4) With the calculated coordinates $X_{E i}, Y_{E i}$, the current value of the shaper outer circle radius $r_{a i}$ is defined by equation

$$
r_{a i}=\sqrt{X_{E i}^{2}+Y_{E i}^{2}}
$$

After that the requirement (26) is checked.

$$
r_{a i}>r_{a} \text {, }
$$

in which the value $r_{a}$ is the main parameter value from the step 2.

5) If the inequality (27) is not fulfilled, the current value of $\psi_{i}$ must be initially increased with pitch $\delta=10^{\circ}$, and after that the steps 3 to 5 are repeated until the requirement (27) is fulfilled.

6) The solution interval is specified, after that the alteration pitch of $\psi_{i}$ is decreased to $\delta=1^{\circ}$ and the steps 3 to 5 are repeated.

7) The decrease of $\delta$ and the calculation of the radius $r_{a i}$ by the coordinates $X_{E i}$ и $Y_{E i}$ continue until the desired accuracy of the $r_{a i}$ is fulfilled.

Numerical example. To demonstrate the proposed algorithm, the coordinates $X_{E}$ и $Y_{E}$ are calculated by Microsoft Excel (Table 1) with the following parameters: $b=10 \mathrm{~mm}, R=31 \mathrm{~mm}, r=28 \mathrm{~mm}, z=8$ and $z g=5$.

The intermediate calculations, related to current parameter $\psi_{i}$ and the radius $r_{a i}$ are situated in 10 rows and 12 columns in the bottom part of the table. The current values of $\psi_{i}$ are set in the odd columns and the current values of $r_{a i}$, calculated by equation (26) are set in the even columns. In that way, the set values of $\psi_{i}$ increase from the top to the bottom and the calculated values of $r_{a i}$ decrease in the same direction. The current values of $X_{E i}$ and $Y_{E i}$ through which $r_{a i}$ is defined, are calculated by the first two equation from the system (25). For clarity, these values are not shown in separated columns.

The calculation of the current values of $r_{a i}$ in the different odd columns are made with different pitch of the parameter $\psi_{i}$. Also. the pitch for increasing $\psi_{i}$ in the odd columns is set in the following way: column $1-$ $10^{0}$; column $3-1^{0}$; column $5-0,1^{0}$; column $7-0,01^{0}$; column $9-0,001^{0}$; column $11-0,0001^{0}$ etc.

The successive increase of the parameter $\psi_{i}$ in the corresponding column continues until the requirement (27) is fulfilled. After that the rest of the rows in this column and next one remain blank. In this way, the current solution in every even column is between the last two rows, in which there are values for radius $r_{a i}$. Thus, the initial value of $\psi_{i}$ from the current odd column in row 1 , is the second to last value from the previous odd column.

Finally the numerical solution is as follows:

- the final value of $\psi$ is defined as an average of the last two values, i.e.

$$
\psi=(4.0667+4.0668) / 2=4.06675
$$

and it is written as a calculated parameter.

- the coordinates $X_{E}$ and $Y_{E}$ from the first two equations from (25) are calculated using this value of $\psi$ and are written as a calculated parameters.

$$
X_{E}=2.823182, \quad Y_{E}=0.200721
$$


Table 1. Numerical solution of the coordinate $X_{E}$ and $Y_{E}$ by Microsoft Excel.

\begin{tabular}{|c|c|c|c|c|c|c|c|c|c|c|c|c|}
\hline & \multicolumn{5}{|c|}{ Set parameters } & \multicolumn{4}{|c|}{ Meshing parameters } & \multicolumn{3}{|c|}{ Calculated values } \\
\hline & $b$ & $R$ & $r$ & $z$ & $z_{2}$ & $R s$ & $R g$ & $a$ & $r a$ & $\psi$ & $X_{E}$ & $Y_{E}$ \\
\hline & 10 & 31 & 28 & 8 & 5 & 30.594 & 19.12 & 11.47 & 19.527206 & 4.06675 & 2.823182 & 0.200721 \\
\hline & \multicolumn{12}{|c|}{ Intermediate calculation for clarification of the angular parameter $\psi$} \\
\hline & $\psi_{i}$ & $r_{a i}$ & $\psi_{i}$ & rai & $\psi_{i}$ & $r a i$ & $\psi_{i}$ & rai & $\psi_{i}$ & rai & $\psi_{i}$ & rai \\
\hline 1 & 0 & 19.8 & 0 & 19.76 & 4.0 & 19.532 & 4.00 & 19.5316 & 4.060 & 19.52765 & 4.0660 & 19.527255 \\
\hline 2 & 10 & 19.1 & 1 & 19.71 & 4.1 & 19.525 & 4.01 & 19.5309 & 4.061 & 19.52758 & 4.0661 & 19.527249 \\
\hline 3 & - & - & 2 & 19.66 & - & - & 4.02 & 19.5303 & 4.062 & 19.52752 & 4.0662 & 19.527242 \\
\hline 4 & - & - & 3 & 19.60 & - & - & 4.03 & 19.5296 & 4.063 & 19.52745 & 4.0663 & 19.527235 \\
\hline 5 & - & - & 4 & 19.53 & - & - & 4.04 & 19.529 & 4.064 & 19.52739 & 4.0664 & 19.527229 \\
\hline 6 & - & - & 5 & 19.46 & - & - & 4.05 & 19.5283 & 4.065 & 19.52732 & 4.0665 & 19.527222 \\
\hline 7 & - & - & - & - & - & - & 4.06 & 19.5277 & 4.066 & 19.52726 & 4.0666 & 19.527216 \\
\hline 8 & - & - & - & - & - & - & 4.07 & 19.527 & 4.067 & 19.52719 & 4.0667 & 19.527209 \\
\hline 9 & - & - & - & - & - & - & - & - & - & - & 4.0668 & 19.527202 \\
\hline \multirow[t]{2}{*}{10} & - & - & - & - & - & - & - & - & - & - & - & - \\
\hline & 1 & 2 & 3 & 4 & 5 & 6 & 7 & 8 & 9 & 10 & 11 & 12 \\
\hline
\end{tabular}

In this table made in Microsoft Excel, after the input of the parameters $b, R, r, z, z_{g}$, the other cells are filled automatically. The final solution of the system (25) is in the right top part of the Table 1, where are the cells for $\psi, X_{E}$ and $Y_{E}$.

\section{Interference verification at $z_{g}=z / 2$}

When the number of shaper teeth is twice as small as the number of the splines, the calculations related to the interference are significantly simplified. Moreover, the numerical solution of the interference angle $\mu$ is substituted with an analytical one by an exact equation.

In this special case, the gear shaper contour is transformed in a part of a circle (an arc) [8] with radius $b / 2$, and the radiuses $R_{g}$ and $r_{a}$ are defined by equations

$$
\begin{gathered}
a=R_{g}=R_{S} / 2=0,5 \sqrt{R^{2}-(b / 2)^{2}}, \\
r_{a}=R-a=R-0,5 \sqrt{R^{2}-(b / 2)^{2}}
\end{gathered}
$$

When $z_{g}=z / 2$ the angles $\lambda$ and $\gamma_{g}$ from equation (22) are defined by Fig. 5. As well as in Fig. 4, in this figure the end position of the gear meshing is shown. In it, the shaper tooth peak point and the spline straight profile are contacting in point $E$. The characteristic feature here is that the triangle $O_{g} L O_{s}$ is isosceles $\left(O_{g} L=O_{g} O_{s}=R g\right), \quad$ and $\quad$ as a result $\angle L O_{s} O_{g}=\angle O_{g} L O_{s}=\lambda$. Then, from the triangle $E L O g$ which sides $R g, \quad r a$ and $b / 2$ are known, the angles $\tau$ and $\delta$ are defined by law of cosine

$$
\begin{aligned}
& \cos \tau=\frac{R_{g}{ }^{2}-r_{a}{ }^{2}+(b / 2)^{2}}{R_{g} b}, \\
& \cos \delta=\frac{R_{g}{ }^{2}+r_{a}{ }^{2}-(b / 2)^{2}}{2 R_{g} r_{a}},
\end{aligned}
$$

after that, the angles $\lambda$ and $\gamma_{g}$ are determined by equations



Fig. 5. Determination of the angles $\lambda$ and $\gamma_{g}$ at $z_{g}=z / 2$. 


$$
\begin{gathered}
\lambda=\frac{\pi}{2}-\tau=\frac{\pi}{2}-\arccos \left(\frac{R_{g}{ }^{2}-r_{a}{ }^{2}+(b / 2)^{2}}{R_{g} b}\right), \\
\gamma_{g}=\delta-2 \lambda=\arccos \left(\frac{R_{g}{ }^{2}+r_{a}{ }^{2}-(b / 2)^{2}}{2 R_{g} r_{a}}\right)- \\
-\pi+2 \arccos \left(\frac{R_{g}{ }^{2}-r_{a}{ }^{2}+(b / 2)^{2}}{R_{g} b}\right) .
\end{gathered}
$$

Then from the equations (22) and (23), the generating interference angle is finally defined by

$$
\begin{aligned}
\mu & =\left[\arccos \left(\frac{r^{2}-r_{a}^{2}-a^{2}}{2 r_{a} a}\right)-\gamma_{g}\right] \frac{z_{g}}{z}- \\
& -\arccos \left(\frac{r^{2}-r_{a}^{2}+a^{2}}{2 r a}\right)-\lambda+\arcsin \left(\frac{b}{2 r}\right)
\end{aligned} .
$$

The equation (34) is valid when $z g=z / 2$, and it allows the value of $\mu$ to be directly calculated. For this purpose, the radius $r_{a}$ and the angles $\lambda$ и $\gamma_{g}$ are previously defined by equations (28), (29), (32) and (33).

\section{Results}

One of the main problem which is solved in profiling a gear shapers for internal straight splines is the determination of the number of shaper teeth $z_{g}$

In article [8] the equation for minimal number of shaper teeth $z_{g}$,min is defined. So that $z_{g, \min }$ is a limit, and beyond it the tool teeth contour is a continuous curve. When $z_{g}<z_{g}$,min, the gear shaper is practically unusable because the tool contour is a broken curve.

The research done in this article shows that the maximum number of shaper teeth for cutting internal straight splines is limited by the impossibility to avoid the interference between the cutting profiles and the cut off profiles. For defining the maximum number of the shaper teeth for cutting standard straight splines [16] the necessary calculations are done. The results for light, medium and heavy series of the splines are shown respectively in Table 2, Table 3 and Table 4 . The presence or absence of interference is determined by the respective values of the interference angle $\mu$ and the level of interference $\Delta$.

In the tables below, the values of $\mu$ and $\Delta$ for every spline opening (with parameters $z, d=2 r, D=2 R$ and $b$ ), are calculated for three or four consecutive values of the number of shaper teeth $z_{g}$.

The Tables 2 and 3 show that when the number of the splines from the light and medium series is $z=6$, the maximum number of the shaper teeth is $z_{g \text {, } \max }=4$, i.e.

$$
z_{g, \max }=z / 2+1 \quad \text { at } \quad z=6 .
$$

Similar to this is the case, when the number of the splines from the light and the medium series is $z=8$.

\begin{tabular}{|c|c|c|c|c|c|c|c|}
\hline \multirow{2}{*}{$\begin{array}{c}z \times d \times 1 \times b \\
(z \times 2 r \times 2 R \times 1)\end{array}$} & \multirow{2}{*}{$z g \mid$} & $\mu$ & $\Delta$ & \multirow{2}{*}{  } & \multirow{2}{*}{$z g$} & $\mu$ & $\Delta$ \\
\hline & & deg & $\mathrm{mm}$ & & & deg & $\mathrm{mm}$ \\
\hline \multirow{3}{*}{$6 \times 23 \times 26 \times 6$} & 3 & 1.05 & 0.211 & \multirow{3}{*}{$6 \times 26 \times 30 \times 6$} & 3 & 1.20 & 0.271 \\
\hline & 4 & 0.38 & 0.076 & & 4 & 0.27 & 0.059 \\
\hline & 5 & -0.93 & $\mid-0.187$ & & 5 & -1.52 & -0.343 \\
\hline \multirow{3}{*}{$6 \times 28 \times 32 \times 7$} & 3 & 1.15 & 0.281 & \multirow{3}{*}{$8 \times 32 \times 36 \times 6$} & 4 & 0.83 & 0.233 \\
\hline & 4 & 0.35 & 0.084 & & 5 & 0.33 & 0.092 \\
\hline & 5 & -1.20 & $\mid-0.293$ & & 6 & -0.37 & -0.104 \\
\hline \multirow{3}{*}{$8 \times 36 \times 40 \times 7$} & 4 & 0.75 & 0.234 & \multirow{3}{*}{$8 \times 42 \times 46 \times 8$} & 4 & 0.62 & 0.225 \\
\hline & 5 & 0.33 & 0.106 & & 5 & 0.30 & 0.109 \\
\hline & 6 & -0.25 & -0.076 & & 6 & -0.15 & -0.057 \\
\hline \multirow{3}{*}{$8 \times 46 \times 50 \times 9$} & 4 & 0.55 & 0.223 & \multirow{3}{*}{$8 \times 52 \times 58 \times 10$} & 4 & 077 & 0.351 \\
\hline & 5 & 0.28 & 0.117 & & 5 & 0.33 & 0.152 \\
\hline & 6 & -0.10 & -0.039 & & 6 & -0.29 & -0.127 \\
\hline \multirow{3}{*}{$8 \times 56 \times 62 \times 10$} & 4 & 0.68 & 0.332 & \multirow{3}{*}{$8 \times 62 \times 68 \times 12$} & 4 & 0.63 & 0.341 \\
\hline & 5 & 0.28 & 0.138 & & 5 & 0.25 & 0.167 \\
\hline & 6 & -0.27 & -0.132 & & 6 & -0.15 & -0.084 \\
\hline \multirow{4}{*}{$10 \times 72 \times 78 \times 12$} & 5 & 0.48 & 0.305 & \multirow{4}{*}{$10 \times 82 \times 88 \times 12$} & 5 & 0.38 & 0.277 \\
\hline & 6 & 0.27 & 0.172 & & 6 & 0.20 & 0.149 \\
\hline & 7 & 0.02 & 0.009 & & 7 & -0.02 & -0.006 \\
\hline & 8 & -0.37 & -0.230 & & 8 & -0.31 & -0.232 \\
\hline \multirow{4}{*}{$10 \times 92 \times 98 \times 14$} & 5 & 0.35 & 0.277 & \multirow{4}{*}{$10 \times 102 \times 108 \times 16$} & 5 & 0.31 & 0.275 \\
\hline & 6 & 0.20 & 0.160 & & 6 & 0.18 & 0.167 \\
\hline & 7 & 0.02 & 0.017 & & 7 & 0.03 & 0.036 \\
\hline & 8 & -0.23 & \begin{tabular}{|l|}
-0.191 \\
\end{tabular} & & 8 & -0.18 & -0.158 \\
\hline \multirow{4}{*}{$10 \times 112 \times 120 \times 18$} & 5 & 0.40 & 0.387 & \multirow{4}{*}{$10 \times 112 \times 120 \times 18$} & 5 & 0.40 & 0.387 \\
\hline & 6 & 0.23 & 0.224 & & 6 & 0.23 & 0.224 \\
\hline & 7 & 0.03 & 0.026 & & 7 & 0.03 & 0.026 \\
\hline & 8 & -0.27 & -0.265 & & 8 & -0.27 & -0.265 \\
\hline
\end{tabular}
Then $z_{g, \max }=5$, where

$$
z_{g, \max }=z / 2+1 \quad \text { at } \quad z=8 .
$$

\begin{tabular}{|c|c|c|c|c|c|c|c|}
\hline \multirow{2}{*}{$\begin{array}{c}z \times d \times D \times b \\
(z \times 2 r \times 2 R \times x)\end{array}$} & \multirow{2}{*}{$z g$} & $\mu$ & $\Delta$ & \multirow{2}{*}{$\mid \begin{array}{c}z \times d_{\times} D \times \\
\left(z \times 2 r_{x} 2 R_{\times} b\right)\end{array}$} & \multirow{2}{*}{$z g$} & \multirow{2}{*}{$\frac{\mu}{\operatorname{deg}}$} & \multirow{2}{*}{$\frac{\Delta}{\mathrm{mm}}$} \\
\hline & & deg & $\mathrm{mm}$ & & & & \\
\hline \multirow{3}{*}{$6 \times 11 \times 14 \times 3$} & 3 & 2.48 & 0.238 & \multirow{3}{*}{$6 \times 13 \times 16 \times 3.5$} & 3 & 2.07 & 0.234 \\
\hline & 4 & 0.22 & 0.021 & & \begin{tabular}{|l|l|}
4 \\
\end{tabular} & 0.33 & \begin{tabular}{|l|l}
0.037 \\
\end{tabular} \\
\hline & 5 & -4.57 & -0.439 & & \begin{tabular}{|l|}
5 \\
\end{tabular} & -3.20 & -0.364 \\
\hline \multirow{3}{*}{$6 \times 16 \times 20 \times 4$} & 3 & 2.13 & 0.297 & \multirow{3}{*}{$6 \times 18 \times 22 \times 5$} & 3 & 2.02 & 0.318 \\
\hline & 4 & 0.13 & 0.018 & & \begin{tabular}{|l|l}
4 \\
\end{tabular} & 0.4 & 0.063 \\
\hline & 5 & -3.97 & -0.554 & & \begin{tabular}{|l|l|}
5 \\
\end{tabular} & -2.90 & -0.455 \\
\hline \multirow{3}{*}{$6 \times 21 \times 25 \times 5$} & 3 & 1.55 & 0.283 & \multirow{3}{*}{$6 \times 23 \times 28 \times 6$} & 3 & 1.90 & 0.380 \\
\hline & 4 & 0.23 & 0.043 & & \begin{tabular}{|l|}
4 \\
\end{tabular} & 0.30 & 0.061 \\
\hline & 5 & -2.32 & -0.424 & & \begin{tabular}{|l|}
5 \\
\end{tabular} & -2.88 & -0.578 \\
\hline \multirow{3}{*}{$6 \times 26 \times 32 \times 6$} & 3 & 1.85 & 0.421 & \multirow{3}{*}{$6 \times 28 \times 34 \times 7$} & 3 & 1.82 & 0.444 \\
\hline & 4 & 0.07 & 0.014 & & \begin{tabular}{|l|}
4 \\
\end{tabular} & 0.25 & 0.060 \\
\hline & 5 & -3.50 & -0.795 & & \begin{tabular}{|l|l|}
5 \\
\end{tabular} & -2.87 & -0.701 \\
\hline \multirow{3}{*}{$8 \times 32 \times 38 \times 6$} & 4 & 1.28 & 0.360 & \multirow{3}{*}{$8 \times 36 \times 42 \times 7$} & 4 & 1.17 & 0.367 \\
\hline & 5 & 0.32 & 0.090 & & \begin{tabular}{|l|}
5 \\
\end{tabular} & 0.37 & 0.116 \\
\hline & 6 & -1.00 & -0.278 & & \begin{tabular}{|l|}
6 \\
\end{tabular} & -0.72 & -0.226 \\
\hline \multirow{3}{*}{$8 \times 42 \times 48 x 8$} & 4 & 0.98 & 0.358 & \multirow{3}{*}{$8 \times 46 \times 54 \times 9$} & 4 & 1.23 & 0.493 \\
\hline & 5 & 0.35 & 0.127 & & \begin{tabular}{|l|}
5 \\
\end{tabular} & 0.37 & 0.150 \\
\hline & 6 & -0.50 & -0.186 & & 6 & -0.78 & -0.317 \\
\hline \multirow{3}{*}{$8 \times 52 \times 60 \times 10$} & 4 & 1.07 & 0.483 & \multirow{3}{*}{$8 \times 56 \times 65 \times 10$} & 4 & 1.07 & 0.518 \\
\hline & 5 & 0.37 & 0.163 & & \begin{tabular}{|l|}
5 \\
\end{tabular} & 0.30 & 0.143 \\
\hline & 6 & -0.60 & -0.273 & & \begin{tabular}{|l|}
6 \\
\end{tabular} & -0.75 & -0.365 \\
\hline \multirow{3}{*}{$8 \times 62 \times 72 \times 12$} & 4 & 1.13 & 0.609 & \multirow{3}{*}{$10 \times 72 \times 82 \times 12$} & 5 & 0.87 & 0.544 \\
\hline & 5 & 0.37 & 0.198 & & \begin{tabular}{|l|}
6 \\
\end{tabular} & 0.38 & 0.238 \\
\hline & 6 & -0.67 & -0.362 & & \begin{tabular}{|l|}
7 \\
\end{tabular} & -0.20 & -0.130 \\
\hline \multirow{3}{*}{$10 \times 82 \times 92 \times 12$} & 5 & 0.68 & 0.492 & \multirow{3}{*}{$10 \times 92 \times 102 \times 14$} & 5 & 0.62 & 0.501 \\
\hline & 6 & 0.28 & 0.200 & & 6 & 0.28 & 0.229 \\
\hline & 7 & -0.2 & -0.148 & & \begin{tabular}{|l|}
7 \\
\end{tabular} & -0.12 & -0.095 \\
\hline \multirow{3}{*}{$10 \times 102 \times 112 \times 16$} & 5 & 0.57 & 0.504 & \multirow{3}{*}{$10 \times 112 \times 125 \times 18$} & 5 & 0.70 & 0.679 \\
\hline & 6 & 0.28 & 0.251 & & \begin{tabular}{|l|}
6 \\
\end{tabular} & 0.32 & \begin{tabular}{|l|l}
0.317 \\
\end{tabular} \\
\hline & 7 & -0.07 & -0.052 & & 7 & -0.12 & -0.117 \\
\hline
\end{tabular}

Table 2. Maximum number of the shaper teeth for cutting standard straight splines - light series.

Table 3. Maximum number of the shaper teeth for cutting 
Table 4. Maximum number of the shaper teeth for cutting standard straight splines - heavy series.

\begin{tabular}{|c|c|c|c|c|c|c|c|}
\hline \multirow{2}{*}{$\begin{array}{c}z \times d \times D \times b \\
(z \times 2 r \times 2 R \times b)\end{array}$} & \multirow[b]{2}{*}{$z g$} & $\mu$ & $\Delta$ & \multirow{2}{*}{$\begin{array}{c}z \times d \times D \times \\
(z \times 2 r \times 2 R \times b)\end{array}$} & \multirow[b]{2}{*}{$z_{\varepsilon}$} & \multirow{2}{*}{\begin{tabular}{|c|}
$\mu$ \\
$\operatorname{deg}$
\end{tabular}} & \multirow{2}{*}{$\frac{\Delta}{\mathrm{mm}}$} \\
\hline & & deg & $\mathrm{mm}$ & & & & \\
\hline \multirow{3}{*}{$10 \times 16 \times 20 \times 2.5$} & \begin{tabular}{|l|l|}
5 \\
\end{tabular} & $\begin{array}{l}1.48 \\
\end{array}$ & 0.207 & \multirow{3}{*}{$10 \times 18 \times 23 \times 3$} & 5 & \begin{tabular}{|l|l}
1.73 \\
\end{tabular} & 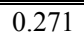 \\
\hline & 6 & 0.28 & 0.040 & & 6 & 0.33 & 0.052 \\
\hline & \begin{tabular}{|l|}
7 \\
\end{tabular} & -1.17 & -0.163 & & 7 & -1.37 & -0.215 \\
\hline \multirow{3}{*}{$10 \times 21 \times 26 \times 3$} & \begin{tabular}{|l|}
5 \\
\end{tabular} & 1.32 & 0.242 & \multirow{3}{*}{$10 \times 23 \times 29 \times 4$} & \begin{tabular}{|l|}
5 \\
\end{tabular} & 1.68 & 0.338 \\
\hline & 6 & 0.20 & 0.036 & & 6 & 0.42 & 0.084 \\
\hline & \begin{tabular}{|l|}
7 \\
\end{tabular} & -1.17 & -0.213 & & \begin{tabular}{|l|}
7 \\
\end{tabular} & -1.12 & -0.225 \\
\hline \multirow{3}{*}{$10 \times 26 \times 32 \times 4$} & \begin{tabular}{|l|}
5 \\
\end{tabular} & 1.37 & 0.309 & \multirow{3}{*}{$10 \times 28 \times 35 \times 4$} & \begin{tabular}{|l|}
5 \\
\end{tabular} & 1.38 & 0.337 \\
\hline & \begin{tabular}{|l|}
6 \\
\end{tabular} & 0.30 & 0.067 & & 6 & 0.17 & 0.042 \\
\hline & \begin{tabular}{|l|}
7 \\
\end{tabular} & -1.00 & -0.226 & & 7 & -1.28 & -0.315 \\
\hline \multirow{3}{*}{$10 \times 32 \times 40 \times 5$} & \begin{tabular}{|l|}
5 \\
\end{tabular} & 1.48 & 0.415 & \multirow{3}{*}{$10 \times 36 \times 45 \times 5$} & \begin{tabular}{|l|l|}
5 \\
\end{tabular} & 1.35 & 0.423 \\
\hline & 6 & 0.28 & 0.080 & & 6 & 0.13 & 0.043 \\
\hline & \begin{tabular}{|l|}
7 \\
\end{tabular} & -1.17 & -0.326 & & \begin{tabular}{|l|}
7 \\
\end{tabular} & -1.33 & -0.416 \\
\hline \multirow{3}{*}{$10 \times 42 \times 52 \times 6$} & \begin{tabular}{|l|l|}
5 \\
\end{tabular} & 1.32 & 0.483 & \multirow{3}{*}{$10 \times 46 \times 56 \times 7$} & \begin{tabular}{|l|}
5 \\
\end{tabular} & 1.27 & 0.512 \\
\hline & \begin{tabular}{|l|}
6 \\
\end{tabular} & 0.20 & 0.071 & & 6 & 0.30 & 0.119 \\
\hline & \begin{tabular}{|l|}
7 \\
\end{tabular} & -1.17 & -0.426 & & 7 & -0.88 & -0.354 \\
\hline \multirow{3}{*}{$16 \times 52 \times 60 \times 5$} & \begin{tabular}{|l|}
8 \\
\end{tabular} & 0.63 & 0.285 & \multirow{3}{*}{$16 \times 56 \times 65 \times 5$} & \begin{tabular}{|l|l|} 
\\
\end{tabular} & 0.62 & 0.300 \\
\hline & \begin{tabular}{|l|}
9 \\
\end{tabular} & 0.25 & 0.115 & & \begin{tabular}{|l|}
9 \\
\end{tabular} & 0.22 & 0.105 \\
\hline & 10 & -0.15 & -0.065 & & \begin{tabular}{|l|}
10 \\
\end{tabular} & -0.22 & -0.103 \\
\hline \multirow{3}{*}{$16 \times 62 \times 72 \times 6$} & \begin{tabular}{|l|}
8 \\
\end{tabular} & 0.67 & 0.357 & \multirow{3}{*}{$16 \times 72 \times 82 \times 7$} & \begin{tabular}{|l|}
8 \\
\end{tabular} & 0.57 & 0.360 \\
\hline & \begin{tabular}{|l|}
9 \\
\end{tabular} & 0.1 & 0.141 & & \begin{tabular}{|l|l|}
9 \\
\end{tabular} & 0.25 & 0.159 \\
\hline & 10 & -0.17 & -0.090 & & 10 & -0.08 & -0.056 \\
\hline \multirow{3}{*}{$20 \times 82 \times 92 \times 6$} & 10 & 0.4 & 0.284 & \multirow{3}{*}{$20 \times 92 \times 102 \times 7$} & 10 & 0.37 & 0.294 \\
\hline & 11 & 0.18 & 0.131 & & 11 & 0.18 & 0.149 \\
\hline & 12 & -0.03 & -0.029 & & 12 & 0.00 & -0.001 \\
\hline \multirow{4}{*}{$20 \times 102 \times 115 \times 8$} & 10 & 0.43 & 0.391 & \multirow{4}{*}{$20 \times 112 \times 125 \times 9$} & 10 & 0.42 & 0.400 \\
\hline & 11 & 0.22 & 0.189 & & 11 & 0.22 & 0.207 \\
\hline & 12 & -0.03 & -0.022 & & 12 & 0.00 & 0.005 \\
\hline & & & & & 13 . & -0.22 & -0.212 \\
\hline
\end{tabular}

When the number of the splines is $z=10$, Table. 2 shows that in the light series $z_{g, \max }=7$. This means

$$
z_{g, \max }=z / 2+2 \text { at } z=10 \text { - light series. }
$$

For the same number of the splines $z=10$ in medium and heavy series (Table. 3 and Table. 4 ) $z_{g, \max }=6$, i.e.

$$
z_{g, \max }=z / 2+1 \text { at } z=10-\text { medium and heavy series. }
$$

Table 4 shows that in the heavy series, when $z=16$ and $z=20$, the maximum number of the shaper teeth is respectively $z g, \max =9$ and $z g, \max =11$. In this case this means that

$$
z_{g, \max }=z / 2+1 \text { at } z=16 \text { and } z=20 .
$$

The summary of the results in Tables 2, 3 and 4 allows to determine the following limitative requirement: the maximum number of the shaper teeth for cutting all standard internal straight splines, except the light series splines with $z=10$, is $z_{g \text {, } \max }=z / 2+1$.

Also, from the results in the applied tables it is clear that the interference depends directly on the splines' height. In this connection, the possibility of interference is greater when higher splines are being cut. This is shown in the Table 2 and Table 3, when the number of the splines is $z=10$. For example, at the $10 \times 92 \times 98 \times 14$ splines with height $h_{1}=r-R_{1}=3 \mathrm{~mm}, z_{g}$,max $=7$, and at the $10 \times 92 \times 102 \times 14$ splines with height $h_{2}=r-R_{2}=5 \mathrm{~mm}, z_{g, \max }=6$. In the case at hand, the parameters $z=10, r=46$ and $b=14$ are the same for the both type of splines. Only the radiuses $R$ of the outer circles $\left(R_{1}=49, R_{2}=51\right)$ are different, as a result the splines height are also different $\left(h_{1} \neq h_{2}\right)$.

For the determination of $z_{g, \max }$ it is necessary that the respective values of the level of interference $\Delta$ to be the smallest positive numbers. When the values of $\Delta$ are negligible small negative numbers, it can be accepted that there is no interference. In these cases the cropping of the straight profile is on the order of some micrometers, which is in the producing tolerance. For example at the $20 \times 92 \times 102 \times 7$ splines in Table. 4 , the theoretical value is $z_{g, \max }=11$, but practically can be accepted $z_{g, \max }=12$ despite the negative value of $\Delta$. In this case the level of interference is $\Delta=-0.001 \mathrm{~mm}$, i.e. too small negative number.

\section{Conclusions}

The research above allows us to draw the following conclusions:

1. The generating interference, when gear shaper and splines are being meshed, the so-called zone of possible interference occurs, which is placed out of the formation zone of the straight splines profile.

2. The interference between two meshed profiles is greater at the position where the peak point of the shaper tooth lies on the splines inner circle $r$

3. Generally, when $z g \neq z / 2$ and the splines centroidal radius $R_{S}$ is defined by equation (3), the level of interference $\Delta$ is determined by numerical solution.

4. In the special private solution, when $z_{g}=z / 2$, the level of interference is defined by equations (24) and (34), independently of the chosen radius of the splines centroidal circle.

5. When the number of the shaper teeth is twice as small as the number of splines that are being cut, the shaper tooth profile and the straight splines profile do not interfere each other.

6. When cutting standard internal straight splines, the maximum number of the shaper teeth with no interference is most often equal to half of the splines plus one. $\left(z_{g, \max }=z / 2+1\right)$.

7. The level of interference depends directly on the set height of the splines that are being cut. Moreover, the increase of the height of the splines, leads to an increase of the unfavorable effect of interference.

The study was supported by contract of University of Ruse "Angel Kanchev", № BG05M2OP001-2.009-0011-C01, " Support for the development of human resources for research and innovation at the University of Ruse "Angel Kanchev". The project is funded with support from the Operational Program " Science and Education for Smart Growth 2014 - 
2020" financed by the European Social Fund of the European Union.

\section{References}

1. D.A. Stephenson, J.S. Agapiou, Metal Cutting Theory and Practice (Third Edition, CRC Press, Taylor \& Francis Group, 2016)

2. B.L. Juneja, G.S. Sekhon, N. Seth, Fundamentals of Metal Cutting and Machine Tools (New Age International Publishers, New Delhi, 2005)

3. S.P. Radzevich, Gear Cutting Tools - Fundamentals of Design and Computation (CRC Press, Boca Raton, Florida, 2010)

4. T. Maeno, K. Mori, Y. Ichikawa, M. Sugawara, Use of liquid lubricant for backward extrusion of cup with internal splines using pulsating motion, J. Mater. Process. Technol. 244 273-281, (2017) https://doi.org/10.1016/j.jmatprotec.2017.02.001

5. R.S. Lee, J.J. Sheu, Y.J. Gau, Optimum die-surface design of gear-spline extrusion using a general surface model, J. Mater. Process. Technol. 28 (3) 365-382, (1991) https://doi.org/10.1016/0924-0136(91)90144-4

6. A.A, Abd-Eltwab, S.Z. El-Abden, I.E. Achmed Khaled, M.N El-Sheikh, R.K. Abdel-Magied, An investigation into forming internally-spline sleeves by ball spinning. Int. J. of Mech. Sci. 134, 399-410, (2017) https://doi.org/10.1016/j.ijmecsci.2017.10.033

7. Q. Zhang, D. Mu, K. Jin, Y. Liu, Recess swaging method for manufacturing the internal helical splines, J. Mater. Process. Technol. 214 (12) 2971 2984, (2014) https://doi.org/10.1016/j.jmatprotec.2014.07.004.
8. O. Alipiev, S. Marinov, T. Uzunov, Optimal tooth profile design of a gear shaper cutter when meshing with internal straight splines. Mech. Mach. Theory 129, 70-79, (2018) https://doi.org/10.1016/j.mechmachtheory.2018.07.010

9. S. Marinov, O. Alipiev, T. Uzunov, Profiling gear chapers for producing straight internal splines. Mechanics of Machines, 120, 21-25, (2018), (in Bulgarian)

10. J.R. Colbourne, The Geometry of Involute Gears (Berlin: Springer-Verlag, New York, 1987)

11. O. Alipiev, S. Antonov, T. Grozeva, Generalized model of undercutting of involute spur gears generated by rack-cutters. Mech. Mach. Theory, 64, 39-52, (2013) https://doi.org/10.1016/j.mechmachtheory.2013.01.012

12. O. Alipiev, Geometric design of involute spur gear drives with symmetric and asymmetric teeth using the Realized Potential Method, Mech. Mach. Theory, 46, 10-32, (2011) https://doi.org/10.1016/ j.mechmachtheory.2010.09.001

13. P. Nenov, B. Kaloyanov, E. Angelova, Geometrical blocking contours as an instrument for hightechnology design of geometry of involute external and internal gear drives, Mechanisms and Machine Science. Power Transmissions 379-388, (2013)

14. F.L. Litvin, A. Fuentes, Gear geometry and applied theory (Cambridge University Press, Cambridge, 2004).

15. F.L. Litvin, Theory of Gearing, (Nauka, Moscow, 1968), (in Russian)

16. ISO 14-1982, Straight-sided splines for cylindrical shafts with internal centering - Dimensions, tolerances and verification, International Organization for Standardization, Geneva, Switzerland, (1982) 